\title{
Religión y política del poder en el mundo Musulmán del Sudeste Asiático ${ }^{1}$
}

Sharon SiddiQue

DeCISION Advisors, SingAPUR

En el mundo musulmán del Sudeste Asiático se observan dos tendencias políticas. La primera, es un islamismo estrictamente fundamentalista, que predica la visión utópica de una comunidad musulmana global (ummah). En ella, la religión triunfa sobre la Política del Poder o Realpolitik. La segunda, aboga por un Islam moderado, que reconoce la diversidad de la ummah y la Realpolitik se adapta a la religión. Antes del 11 de septiembre, los fundamentalistas controlaron el debate moral, mientras los moderados se encontraban a la defensiva. La guerra contra el terrorismo ha invertido los papeles, ya que ahora los moderados tienen la oportunidad de fijar la agenda musulmana.

\section{DOS TENDENCIAS POLITICAS}

La prensa mundial está tratando de ponerse al día en su comprensión de las complejidades de la presencia musulmana global, fenómeno recientemente descubierto. El foco de atención ha sido la relación entre Islam y Occidente. Pero los acontecimientos al interior del mundo islámico son igualmente importantes, y es en este aspecto que el Sudeste Asiático tiene algo que decir.

Hace algunos años, un académico musulmán egipcio estaba recorriendo dicha zona. Ante la pregunta si había venido a enseñar, contestó: "No, he venido a aprender. En la historia de nuestra civilización islámica, la renovación se inicia en la periferia y revitaliza el centro." Cabe preguntarse entonces qué lecciones es posible encontrar en el ámbito musulmán del Sudeste Asiático.

En el siglo XX surgieron dos variantes definidas en el mundo político musulmán. La primera es un Islam estricto, fundamentalista, que disemina una visión utópica de una comunidad musulmana global (ummah). La segunda es un Islam moderado que reconoce la diversidad de la ummah. En la primera, la religión triunfa sobre la Realpolitik. En la segunda, la Realpolitik se adapta a la religión.

Antes del 11 de septiembre, los fundamentalistas controlaron el debate moral, mientras los moderados se encontraban a la defensiva. La guerra contra el terrorismo ha invertido los papeles, y ahora los moderados tienen la oportunidad de fijar la agenda musulmana. Los musulmanes moderados en el Sudeste Asiático están en una sólida posición de capitalizar esta situación a su favor, porque han desarrollado una base de legitimidad política. Aunque la islamización ha sido una 
fuerza poderosa, los musulmanes moderados han logrado en general controlar el desafío fundamentalista.

El impacto visible del 11 de septiembre en la política nacional ha sido especialmente intenso en Malasia. El partido UMNO (Organización Nacional de Malayos Unidos) parece imponerse sobre el PAS (Partia Islam Se-Malaysia). Antes del 11 de septiembre, impulsado por avances electorales y liderando una coalición multiracial alternativa, Barisan Alternativa (BA), el PAS se encontraba a la ofensiva.

Después del 11 de septiembre, el PAS ha sido puesto a la defensiva. El BA sufrió un duro golpe con la salida del Partido de Alianza Democrática (DAP), basado en la etnia china. El líder del UMNO, el Primer Ministro Mahathir, anunció que Malasia ya constituye un "estado islámico", dejando al PAS la carga de probar que no sería así. Esta tendencia parece confirmarse con la victoria del Frente Nacional (Barisan National, BN) en la elección complementaria en el estado de Perlis, en enero de 2002.

\section{EL LLAMADO DEL PANISLAMISMO}

El fundamentalismo se articula políticamente en el panislamismo. Su principal rasgo distintivo es la realización de la visión islámica a nivel internacional. Los panislamistas recorren el mundo, impartiendo lecciones, escuchando y exhortando a los fieles. A juzgar por su influencia, son capaces, por medio de su mensaje, de trascender las preocupaciones más inmediatas asociadas con las lealtades tribales, étnicas y nacionales. Las siguientes tres razones explicarían su éxito:

- el atractivo de una identidad global alternativa.

- la naturaleza utópica de la experiencia personal.

- la promesa de un orden social integral y equitativo.

El panislamismo ofrece a los musulmanes una identidad alternativa. Los musulmanes en el Sudeste Asiático son parte integral de la ummah (comunidad musulmana), que es una de las comunidades globales más desarrolladas del mundo. La ummah se comunica en un lenguaje común, el árabe. Comparte las mismas tradiciones. Celebra los mismos rituales. La identificación con más de mil millones de musulmanes en todo el mundo es motivo de orgullo. Alrededor de $20 \%-22$ millones- viven en el Sudeste Asiático.

Esta identidad se fortalece a través de experiencias personales. Primero, la tradición islámica, compleja y rica en tradiciones, refuerza la solidaridad de la ummah. Ello ocurre, por ejemplo, en las cinco oraciones diarias (salat), que todo musulmán debe realizar cinco veces al día, en árabe, orientado hacia La Meca. Una segunda experiencia es la de compartir anualmente los rigores del mes de ayuno, el Ramadán. En tercer lugar, está la educación religiosa. Las escuelas religiosas en el Sudeste Asiático dan gran importancia al dominio del árabe y a la especialización en aspectos canónicos. Miles de musulmanes estudian en los centros islámicos en el Medio Oriente y el Sur de Asia.

Una visita a La Meca, la ciudad santa del Islam, es una experiencia intensamente personal. Cada año, dos millones de miembros de la ummah hacen el peregrinaje (haj) a La Meca, donde todos 
son iguales, cada uno ante Dios. Alrededor de 10\%-doscientos mil- de los participantes en la haj provienen del Sudeste Asiático. Debido a la distancia, la mayoría visita el Medio Oriente sólo una vez en su vida. Aquellos que retornan como hajis (los que han realizado el peregrinaje) revitalizan la visión utópica de la fe musulmana. Además, en el contexto de esta experiencia intensamente religiosa, se reverencia a los árabes y a su lengua.

Los mensajeros panislámicos, por lo general, no operan exclusivamente en un marco nacional. Algunos rechazan al estado-nación como una institución occidental. Otros lo consideran simplemente irrelevante para su mensaje. Osama bin Laden, es un hombre que no tiene país. Expulsado de Arabia Saudita, los talibanes le dieron refugio, pero está claro que no lo aceptaron como afgano. En la medida que es un apátrida, puede suscribir una apelación incondicional a la ummah.

Hay ciertos elementos comunes en el mensaje panislámico global. La humanidad se divide simplemente entre musulmanes y no musulmanes (kafir). Este fuerte dualismo se extiende desde la sociedad al territorio. La configuración geográfica del mundo se divide en el territorio del Islam, dar al Islam, y el territorio no musulmán, dar al harb (literalmente, el territorio de la guerra).

Los panislamistas exhortan a los musulmanes a practicar su fe. Esto significa que se trata de llevar a la realidad la visión fundamentalista. Ellos no distinguen entre lo secular y lo sagrado. El Corán y las tradiciones del Profeta Mahoma hadith constituyen la base del orden político, económico, jurídico y social, expresado en la shariah (ley divina). Durante demasiado tiempo, estas prescripciones han sido un factor central de inhibición que ha obstruido el debate abierto entre los musulmanes, así como el diálogo entre musulmanes y no musulmanes. En la medida que no han reconocido la necesidad de conversar en un contexto de diversidad, los musulmanes han debilitado su propio mundo.

\section{LA ADAPTACIÓN DEL ISLAM}

El Sudeste Asiático se caracteriza por una compleja heterogeneidad religiosa, étnica y cultural. El árabe no es la lengua nativa de los musulmanes de la zona, y el Medio Oriente no es su tierra de origen. Históricamente, se ha desarrollado un fuerte sentido de identidad cultural, el que ha resistido la arabización del complejo mosaico cultural del Sudeste Asiático. Ello plantea una interrogante que probablemente podrá encontrar mejores respuestas desde la periferia: cuál es el núcleo de la fe que se practica, y cuáles son los elementos decorativos que se le han agregado. En otras palabras, ¿qué elementos de lo que, en términos generales, llamamos la cultura musulmana, deben ser aceptados como fundamentales para la fe, y cuáles pueden adaptarse a las condiciones locales?

No cabe duda que el Islam en el Sudeste Asiático celebra la diversidad. Esto se confirma a través de su complejo mosaico étnico. El grupo más grande es el javanés, que comprende cerca de 80 millones de personas, principalmente habitantes de J ava central y oriental. No todos los javaneses son musulmanes; algunos son cristianos o hindúes. Los malayos, que suman unos 15 millones, tienen un rol regional muy importante, formando la mayoría de las poblaciones de Malasia y Brunei. En Indonesia, Singapur, Tailandia y Filipinas hay significativas minorías malayas. También hay dos destacadas sociedades matrilineales -en Minangkabau en Indonesia y en Negeri Sembilan en Malasia. 
Las comunidades musulmanas del Sudeste Asiático han gozado tradicionalmente de un alto grado de armonía interétnica e interreligiosa, así como de igualdad de género. Las mujeres musulmanas han tenido papeles destacados - como reinas en Aceh en el siglo XV, como hábiles diplomáticas en Riau en el siglo XVIII, como agricultoras, comerciantes y profesoras de religión. Los musulmanes han convivido armónicamente durante siglos con budistas, cristianos y otros. Los movimientos musulmanes militantes no son la norma, sino excepciones históricas.

Los individuos ostentan estas identidades múltiples de manera bastante cómoda. Surin Pitsuwan, quien recientemente fuera Canciller de Tailandia, es un político tailandés respetado, un académico musulmán y es dirigente de la comunidad malaya. El destacado intelectual musulmán Nurcholish Madjid es también un nacionalista indonesio declarado. La red IAIN (de instituciones musulmanas de educación terciaria) tiene una matrícula de miles de estudiantes, que reciben una amplia educación en indonesio y árabe.

Los estados del Sudeste Asiático de mayoría musulmana -Brunei, Indonesia y Malasia- tienen también mucho que contribuir a la experiencia del desarrollo institucional. En cada país, la evolución del sistema ha sido única, pero el denominador común sigue siendo la constante presión política para ampliar el rol de la shari'ah.

En Brunei, esto se expresa por medio de la ideología estatal Melayu Islam Beraja (MIB-Sultanato Malayo Musulmán). En Malasia, el problema principal se plantea en relación con la interpretación de la Constitución, especialmente el artículo 3.1, que declara al Islam como religión oficial. En Indonesia ha tenido lugar una batalla de 50 años sobre la inclusión de una frase adicional en el primer principio de la ideología del Estado, Pancasila, que dice "Fe en un Dios Supremo." Algunos políticos musulmanes siguen demandando la inclusión de las palabras "para los musulmanes, es obligatorio observar la shari'ah."

Cuando en un territorio hay una clara mayoría de población musulmana, frecuentemente se expresan aspiraciones de que se gobierne de acuerdo a preceptos islámicos. El deseo de crear "estados islámicos" en el sur de Filipinas, el sur de Tailandia y en Aceh, ejemplifica esta tendencia en el Sudeste Asiático. Aun en países en que no existen movimientos separatistas, como Singapur, se ha procurado lograr un importante grado de adaptación a los requerimientos musulmanes de desarrollar instituciones separadas - para la recolección y distribución de caridad (zakat), la administración del derecho civil musulmán, etc.

La adaptación a estas presiones exige realizar difíciles actos de equilibrio. Tanto Malasia como Indonesia tienen importantes minorías no musulmanas, así como divisiones étnicas. En la medida que la mayor parte de la oposición musulmana a los partidos políticos moderados obtiene su apoyo intelectual del panislamismo, la tarea se vuelve aún más complicada. Solo una pequeña minoría de los musulmanes en Filipinas y Tailandia apoya aspiraciones independentistas. Sin embargo, tales movimientos permiten comprobar las complejidades de la participación en la ummah.

Finalmente, a pesar de los retrocesos económicos desde 1997, las economías (musulmanas) del Sudeste Asiático han tenido un éxito razonable en términos de desarrollo y de integración a la economía global. A partir de los años 70, Indonesia logró durante 30 años alcanzar un crecimiento medio de $7 \%$ anual. Los gobiernos musulmanes del Sudeste Asiático han demostrado la capacidad de promover y negociar el ingreso de inversiones de empresas multinacionales, así como 
de conducir sectores manufactureros desarrollados, orientados a las exportaciones. Es probable que ignorar al Islam en la evaluación de los paradigmas de desarrollo del Sudeste Asiático, haya constituido una omisión.

Malasia es un caso de éxito económico. En Malasia, el partido musulmán gobernante UMNO lidera un Frente Nacional multirracial (Barisan National o BN), el que comprende partidos no musulmanes de bases china e india. BN tiene una mayoría parlamentaria de dos tercios. El gobierno del BN, que ha estado en el poder desde los años 50, exhibe impresionantes logros en materia de desarrollo. Ante esto, cabe preguntarse si Malasia es un caso exitoso de carácter secular o musulmán.

\section{UNA VISIÓN RETROSPECTIVA POST-11 DE SEPTIEMBRE}

Mucho se ha escrito sobre el 11 de septiembre como el toque del despertador para el mundo occidental. También es el toque del despertador para el mundo musulmán. Los fundamentalistas han sido derribados de la tribuna moral. La guerra contra el terrorismo ha invertido los papeles; ahora los moderados tienen la oportunidad de fijar la agenda musulmana. El desafío es enorme. Los musulmanes moderados deben reorientarse inmediatamente. Deben mirar hacia el interior del mundo musulmán, y reorientar su posición a nivel global. También deben mirar hacia afuera, y fortalecer un diálogo con los no musulmanes.

Antes del 11 de septiembre, los musulmanes en el Sudeste Asiático vivían en un mundo islámico poco vinculado con su entorno. Rara vez se reconocían las diferencias al interior de la ummah, 0 al menos, éstas no se trataban abiertamente. Aún más escasos eran los diálogos con no musulmanes acerca de estos temas. Después de esa fecha, los musulmanes de la región sienten el llamado a tomar partido y elegir opciones.

La reacción inmediata de los musulmanes del Sudeste Asiático fue distanciarse de los espantosos actos de violencia de Nueva York y Washington, y condenar a sus perpetradores. Sin embargo, a partir del bombardeo de Afganistán, las opiniones musulmanes han cristalizado en dos posiciones opuestas sobre la guerra antiterrorista, la posición de apoyo y la de crítica.

El apoyo - algunas veces muy condicionado- proviene de los gobernantes de los estados. Los críticos se inspiran en el panislamismo. La gran mayoría guarda silencio. Los musulmanes del Sudeste Asiático se encuentran en una encrucijada. El camino que escogerá la mayoría en los próximos meses y años influirá la orientación futura de la región.

Los líderes políticos del Sudeste Asiático musulmán enfrentan el dilema de escoger entre las ventajas de política exterior de apoyar la coalición conducida por Estados Unidos, y el imperativo interno de expresar solidaridad con los ideales panislamistas. Enfrentan un dilema entre Realpolitik y religión.

Durante una visita a Washington, D.C. que había sido programada antes del 11 de septiembre, la Presidenta Megawati, de Indonesia, fue la primera líder musulmana que comprometió personalmente su apoyo a la guerra contra el terrorismo encabezada por los Estados Unidos. En Malasia, el Primer Ministro Mahathir ha sido consistente en mantener una actitud de reserva. Ha sido especialmente crítico de los bombardeos en Afganistán. Es interesante observar que el mayor apoyo a Estados Unidos en el Sudeste Asiático proviene de la Presidenta Arroyo, de Filipinas, 
quien ha vinculado su propia campaña para someter el movimiento separatista musulmán en el sur de su país con la guerra global contra el terrorismo.

Los no musulmanes de la región han aprovechado rápidamente la oportunidad para ampliar este debate musulmán al plano de un diálogo nacional. Antes del 11 de septiembre, comentar los asuntos musulmanes era algo estrictamente reservado a los musulmanes. En Malasia, por ejemplo, la rama femenina de la Asociación Malasia China (MCA) recientemente organizó un seminario destinado a entender el Islam político. Fue un evento inédito, que ha sido seguido por otros. Malasia está reafirmando su propia identidad nacional como un modelo de "estado islámico" moderado. El proceso es conducido por el partido oficialista UMNO, que se ha revitalizado, y por el Primer Ministro, que está manteniendo un perfil alto que le permite recuperar popularidad.

Por otra parte, los críticos más explícitos provienen principalmente de organizaciones políticas de oposición, grupos estudiantiles, movimientos religiosos, y grupos de refugiados. La atención de la prensa se ha concentrado abrumadoramente en estos grupos, sobre todo en su retórica antioccidental y sus ataques a la política exterior de Estados Unidos en el Medio Oriente. En general, estos críticos pueden ser descritos como una "masa flotante" -carentes de derechos, alienados, desilusionados, y con débil acceso al poder político nacional.

Los críticos aún deben enfrentar las implicaciones más amplias de lo que los líderes norteamericanos han llamado una guerra internacional y de largo plazo contra el terrorismo. En cambio, los líderes políticos de la región han comenzado a encarar las consecuencias de largo plazo de una campaña, conducida por Estados Unidos, en los ámbitos de la soberanía nacional y la seguridad. ¿Cómo se manejará el equilibrio entre la soberanía nacional y la seguridad internacional? En el Sudeste Asiático, este tema tendría dimensiones bilaterales así como regionales.

LOS miembros de ASEAN han acordado cooperar compartiendo informaciones de inteligencia sobre las actividades de separatistas y elementos radicalizados. Frente a las demandas norteamericanas de cooperación nacional para el seguimiento de vinculaciones con la red Al-Queda u otros grupos terroristas, es probable que se tomen iniciativas regionales que amortiguarían la presión y disminuyan las sensibilidades. El tema de la soberanía nacional parece ser especialmente sensible para los indonesios. Esto se debe a la fluidez de la situación política interna, en la cual los sentimientos anti-norteamericanos tienen más que ver con la falta de avances económicos que con la marea de la islamización.

La matanza del 11 de septiembre ha echado por tierra la imagen generalmente benigna que prevalecía acerca de la construcción panislámica utópica de la ummah, proyecto que se creía limitado a los experimentos de "estado islámico" en países de mayoría musulmana. En adelante, tampoco será posible ignorar que desde ahora, estos modelos panislámicos deberán ser dimensionados en su contexto, sin limitarse a los criterios seculares de evaluación de proyectos de desarrollo. Es necesario contar con paradigmas explícitamente definidos en términos musulmanes. El desafío para los musulmanes moderados es desarrollarlos ahora.

Si fuera correcta la tesis de nuestro académico egipcio, y efectivamente la renovación comienza en la periferia, entonces ciertamente algunos de estos paradigmas deberían surgir a partir de las experiencias únicas del Sudeste Asiático. El primer paso es comenzar a encontrar respuestas a interrogantes críticas, tales como: 
- ¿Por qué son relevantes los ejemplos históricos del Sudeste Asiático?

- ¿Qué es árabe y qué es islámico?

- ¿Qué prácticas tradicionales son compatibles con el Islam?

- ¿Cómo ha tenido lugar la adaptación a la diversidad multirracial y multireligiosa?

- ¿Dónde es posible encontrar modelos de desarrollo apropiados?

- ¿Quién debería ejercer el liderazgo, y cómo debería elegirse a los líderes?

- ¿Cómo deberían enfrentarse las fuerzas de la globalización? 


\section{ESTAD O, INTEGRACIÓN Y MEGATEN DENCIAS CONTEM PORÁNEAS}

Desde mediados de la década de 1990, los gobiernos chilenos y diversas organizaciones de la sociedad civil concentran parte importante de sus esfuerzos en el avance de los procesos de modernización del Estado y de perfeccionamiento del sistema político democrático. En este contexto nacional existe demanda por debates y visiones prospectivas con respecto a las funciones del Estado en el proceso de modernización.

Los trabajos publicados en esta sección constituyen un aporte concreto desde el Instituto de Ciencia Política de la Pontificia Universidad Católica de Chile para la reflexión y el debate sobre las funciones que cabe al Estado en los procesos de modernización. Ellos sintetizan resultados de investigación, en el marco de un estudio encargado por MIDEPLAN en 1998, y que culminó en enero de 1999 con un seminario titulado Estado e Integración Nacional. 